\title{
Prognostic factors for ampullary adenocarcinomas: tumor stage, tumor histology, tumor location, immunohistochemistry and microsatellite instability
}

\author{
Fausto Sessa - Daniela Furlan - Clementina Zampatti • \\ Ileana Carnevali $\cdot$ Francesca Franzi $\cdot$ Carlo Capella
}

Received: 20 March 2007 /Revised: 5 June 2007 / Accepted: 6 June 2007 / Published online: 26 July 2007

(C) Springer-Verlag 2007

\begin{abstract}
Prognostic factors for ampullary carcinomas (ACs) are poorly defined. Fifty three resected ACs were analyzed for CDX2, MUC1, MUC5AC, MUC6, MUC2, and for mismatch repair proteins (hMLH1, hMSH2, PMS2, hMSH6) using immunohistochemistry. Microsatellite instability (MSI) status was evaluated by fluorescently labeled PCR using an automated sequencer. Univariate and multivariate analysis was performed for clinicopathological, immunohistochemical and molecular parameters. CDX2 was found in 32 out of $53(60 \%)$ ACs with a significantly higher frequency among intestinal ACs compared with biliopancreatic (BP) ACs. The MUC1, MUC5AC, MUC6, MUC2 apomucins were expressed in 75, 43, 39, and 28\% of ACs, respectively, with a significantly higher coexpression of MUC1/MUC5AC in BP ACs. MSI and loss of expression of hMLH1/PMS2 or hMSH2/hMSH6 proteins were observed only in intestinal ACs. Factors significantly correlated with improved survival in the univariate analysis were: low stage, absence of lymph nodes metastases, negative
\end{abstract}

Research support: this study has been in part supported by a grant from the University of Insubria, Varese, Italy.

\footnotetext{
F. Sessa · D. Furlan · I. Carnevali · F. Franzi · C. Capella Division of Anatomic Pathology,

Department of Human Morphology, University of Insubria,

Varese, Italy

C. Zampatti

Ospedale Di Circolo,

Busto Arsizio, Varese, Italy

F. Sessa $(\square)$

Department of Pathology, Ospedale di Circolo,

Viale Borri, 57,

21100 Varese, Italy

e-mail: fausto.sessa@uninsubria.it
}

surgical margins ( $R 0$ status), and presence of MSI. In the multivariate analysis, stage was the only independent prognostic factor of survival. We conclude that stage is the only independent prognostic factor of survival in the multivariate analysis, whereas histological criteria and the immunohistochemical expression of apomucins and CDX2 are helpful in the classification and understanding of the histogenesis of ACs.

Keywords Ampulla of Vater - Cancer - Prognostic factor . Microsatellite instability. CDX2

\section{Introduction}

There are different histological classifications of ampullary carcinomas (ACs) which $[2,3,20]$ makes comparison of clinical, therapeutic, and prognostic studies of these tumors difficult. So far, two major histological types of these tumors have been recognized: an intestinal type, arising from intestinal mucosa of the papilla, and a biliopancreatic (BP) type, deriving from the BP ductal epithelium. Nevertheless, conflicting data have been reported about the frequency of these two histological types due to the absence of reliable and consistent histomorphological criteria for differential diagnosis $[2,20,31]$. As patients with intestinal-type ampullary ACs have been reported to have a significantly better prognosis than patients with BP-type ACs [2, 20], the clear differentiation between the two tumor types would have clinical implications.

Although immunohistochemistry has had only limited value to date in unequivocally differentiating BP ACs from intestinal-type ACs, several authors have suggested that knowledge of CDX2 and apomucin expression may be 
useful for this purpose and may have a prognostic value $[10,12,16,18,21,23,26,31]$. In particular, adenocarcinomas of the ampulla of Vater show positive reactivity for MUC1, MUC2, MUC5AC, and MUC6 [16], and some authors suggested that the prognosis of $\mathrm{AC}$ is worse with stronger staining for MUC1 and weaker staining for MUC2 $[21,26]$.

Human CDX2 is a homeobox gene encoding for an intestine-specific transcription factor [7, 14]. The CDX2 protein regulates normal development and differentiation of the small and large intestine and is expressed in the nuclei of epithelial cells throughout the intestinal tract both in embryonic and in postnatal life. Some authors have suggested that the immunohistochemical analysis of CDX2 expression may be a useful marker for identifying intestinaltype carcinomas with a sensitivity and specificity higher than $90 \%$ [5, 30]. In particular, very recently, Hansel et al. [18] reported that CDX2 expression may identify intestinal-type ACs with a favourable prognostic outcome.

Finally, few studies assessed the role of DNA mismatch repair (MMR) in ACs, and discrepant data are available about the frequency of a widespread microsatellite instability (MSI) in these cancers [1, 25, 27]. To date, the largest series of ACs was investigated for MSI status by Scarpa et al. [27] demonstrating the occurrence of MSI in $15 \%$ of cases as well as a significant correlation between MSI and a better 5 year survival in these patients compared with the remaining cases.

The aim of this investigation was to evaluate in a large series of ampullary cancers the prognostic value of clinicopathological, immunohistochemical, and molecular parameters such as location, histology, and stage of tumors, MSI status as well as the immunohistochemical expression of the MUC1, MUC2, MUC5AC, MUC6 apomucins and CDX2 protein.

\section{Materials and methods}

Histological and immunohistochemical study

Fifty-three cases of surgically resected primary carcinomas of the ampulla of Vater (ACs) were retrieved from the surgical pathology files at the Department of Pathology (University of Insubria, Varese, Italy) and the Ospedale di Circolo (Busto Arsizio, Varese, Italy). The selection of the cases was based on the availability of sufficient tumor samples for histopathological, immunohistochemical, and molecular analysis. Moreover, only patients harboring sporadic tumors were eligible for the study (two cases of ampullary cancers were excluded because of their association with familial adenomatous polyposis and with hereditary nonpolyposis colorectal cancer syndrome, respectively).
The ethical consent of each patient was collected according with the rules of the local ethical committee.

Formalin-fixed and paraffin-embedded tissue sections were stained with hematoxylin and eosin (H\&E) and Alcian Blue periodic acid Schiff stain (AB-PAS). The slides were reviewed to confirm the diagnosis according to the WHO classification (2001) and to study the extension of the neoplastic disease [17]. In each tumor, we considered both the main histological pattern (intestinal or BP) and a second or a third pattern when present in more than $10 \%$ of neoplastic tissue according to the criteria reported by Albores-Saavedra et al. [2]. The macroscopic classification as described by Cubilla and Fitzgerald [11] was used to distinguish the tumors as periampullary (PA), intra-ampullary (IA), and mixed types (MT). Follow-up data were collected by contacting clinicians and/or by consulting the Tumor Registry of the Lombardy region (Italy).

Immunoperoxidase studies were performed on sections prepared from formalin-fixed and paraffin-embedded specimens that were dewaxed and rehydrated using Bio-clear (Bio-Optica, Milan, Italy) and graded alcohols. Endogenous peroxidase was blocked by dipping sections in 3\% aqueous hydrogen peroxide for $10^{\prime}$ and antigen retrieval was performed with $10^{\prime}$ microwave treatment in $10-\mathrm{mM}$ citrate buffer, $\mathrm{pH}$ 6. The immunostaining was performed with the avidin-biotin-peroxidase complex technique using diaminobenzidine as a chromogen. The sections were incubated overnight at $4{ }^{\circ} \mathrm{C}$ with mouse monoclonal antibodies against full-length hMLH1 protein (G168-15, PharMingen, San Diego, CA) at 1:100 dilution; carboxy-terminal fragment of hMSH2 protein (FE11, Oncogene Research Products, Cambridge, MA) at 1:200 dilution; hMSH6 protein (44, BD Transduction Laboratories, Europe) at 1:200 dilution; PMS2 (A16-4, BD Biosciences, Europe) at 1:200 dilution. Sections were lightly counterstained with hematoxylin. The normal staining pattern for hMLH1, hMSH2, hMSH6, and PMS2 was nuclear, and a case was considered positive only in the presence of nuclear staining of neoplastic cells. A case was considered negative for expression of hMLH1, hMSH2, hMSH6, and PMS2 when there was a complete absence of nuclear staining of neoplastic cells in the presence of an unquestionable internal positive control, represented by normal epithelial cells, stromal cells, muscle cells, or lymphocytes [8]. Immunoreactive intraepithelial lymphocytes were detected by using a mouse monoclonal antibody anti-CD3 (Biogenex, San Ramon, CA, USA) at 1:100 dilution.

The immunohistochemical study of intestinal transcriptional factor $\mathrm{CDX} 2$ was done with monoclonal antibody CDX2-88 (Biogenex) raised against a full-length CDX2 recombinant protein at 1:100 dilution.

Apomucins were studied using mouse monoclonal antibodies against MUC1 glycoprotein (MA695, Novacastra 
Lab., Newcastle, UK) at 1:100 dilution, MUC2 glycoprotein (CCP58, Novacastra Lab) at 1:200 dilution, MUC5AC glycoprotein (CLH2, Novacastra Lab) at 1:100 dilution, and MUC6 glycoprotein (CLH5, Novacastra Lab) at 1:100 dilution.

All immunostaining results were evaluated according to the percentage of immunopositive tumor cells ranging from less than $10 \%$ of positive cells $(+)$, between 10 and $50 \%$ of positive cells $(++)$ and more than $50 \%$ of positive neoplastic cells $(+++)$. Cases with no immunoreactive cells were indicated as negative $(-)$.

DNA extraction and microsatellite analysis

The MSI status of all 53 ampullary cancers was determined. Tumor DNA was extracted from archival paraffin-embedded tissues using DNeasy Tissue kit (Qiagen, Hilden, Germany) after a manual microdissection to enrich the neoplastic cellularity of at least $80 \%$ in all tumor samples [15]. MSI status was assessed by a fluorescent pentaplex PCR of five quasi-monomorphic mononucleotide repeats (Bat-26, Bat25, NR-21, NR-22, NR-24) according to the amplification conditions reported by Suraweera et al. [28]. The fluorescently labeled PCR products were electrophoresed on an Applied Biosystems 310 automated DNA sequencer (Applied Biosystems, Milan, Italy). This panel of quasi-monomorphic mononucleotide markers can identify the MSI status of tumors without the need for matching normal DNA. However, the size of all PCR products from tumor DNA were always aligned with normal-sized alleles from a germline DNA sample. A tumor was classified as MSI when allelic size variations were observed in $\geq 3$ out of five markers and as MSS when allelic size variations were observed in $\leq 2$ out of the five markers.

\section{Statistical analysis}

The statistical analysis was performed using Fisher's exact test and the $\chi^{2}$ test with Yates' correction (SPSS 7.5 software). The survival analysis was performed by employing the Kaplan-Meier product limit estimate of probability of survival against time, producing a product limit survival curve for each of the following variables: tumor size, macroscopic localization, histological type, grade, stage, presence of lymph node metastases, immunohistochemical expression of CDX2, MUC1, MUC2, MUC5AC, MUC6, and MSI status.

Multivariate analysis was carried out using the Cox proportional hazard model. Variables with $p<0.15$ in the univariate analysis were entered into the multivariate analysis.

For all the survival analyses, the Survan XL program, version 1.14 (copyright $($ 1995-1997), was employed.

\section{Results}

Histopathological study

The series of patients with ACs included 30 men and 23 women with a mean age at diagnosis of 65.8 years (range 44-82 years). The comprehensive results of the clinicopathological and immunohistochemical study of these tumors are reported in Tables 1 and 2, respectively. According to Cubilla and Fitzgerald's criteria, 11 of 53 tumors $(21 \%)$ were classified as PA, 35 of $53(66 \%)$ as IA and 7 of $53(13 \%)$ as MT ACs. Histologically, 23 ACs $(43.4 \%)$ were classified as intestinal (I) type and 30 (56.6\%) as BP type (Fig. 1). A second histological pattern (intestinal or mucinous) was detected in eight cases $(26.6 \%)$

Table 1 Clinicopathological, molecular, and outcome data for ampullary carcinoma

\begin{tabular}{|c|c|c|}
\hline Variable & $N^{\circ}$ & Percent \\
\hline Number of cases & 53 & \\
\hline Mean age (years $\pm \mathrm{SD}$ ) & $65.8 \pm 8.67$ & \\
\hline \multicolumn{3}{|l|}{ Gender } \\
\hline Male & 30 & $(56.6)$ \\
\hline Female & 23 & $(43.4)$ \\
\hline \multicolumn{3}{|c|}{ Macroscopic classification } \\
\hline $\mathrm{PA}$ & 11 & $(20.7)$ \\
\hline IA & 35 & $(66)$ \\
\hline MT & 7 & (13.3) \\
\hline \multicolumn{3}{|l|}{ Histotype } \\
\hline $\mathrm{BP}$ & 30 & $(56.6)$ \\
\hline I & 23 & $(43.4)$ \\
\hline \multicolumn{3}{|l|}{ Grade of differentiation } \\
\hline G2 & 33 & $(62.2)$ \\
\hline G3 & 20 & $(37.8)$ \\
\hline \multicolumn{3}{|l|}{ Staging } \\
\hline I & 18 & $(36.7)$ \\
\hline II & 26 & $(53)$ \\
\hline III & 5 & $(10.3)$ \\
\hline \multicolumn{3}{|l|}{ Lymph nodal metastasis } \\
\hline No & 20 & $(40.8)$ \\
\hline $\mathrm{N}+$ & 29 & $(59.2)$ \\
\hline \multicolumn{3}{|l|}{ Resection margin } \\
\hline R0 & 42 & $(80.7)$ \\
\hline $\mathrm{R}+$ & 10 & (19.3) \\
\hline \multicolumn{3}{|l|}{ MSI status } \\
\hline MSI+ & 5 & $(9.4)$ \\
\hline MSI- & 48 & $(90.4)$ \\
\hline \multicolumn{3}{|l|}{ Follow-up } \\
\hline ANED & 14 & $(27)$ \\
\hline DOD & 29 & $(55.7)$ \\
\hline POD & 9 & $(17.3)$ \\
\hline
\end{tabular}

$I A$ Intra-ampullary, $P A$ periampullary, $M T$ mixed type, $I$ intestinal type, $B P$ biliopancreatic type, $M$ mucinous type, $M e d$, medullary type, $S$ solid type, $n a$ not available, $D O D$ died of disease, $A N E D$ alive with no evidence of disease, $P O D$ postoperative died 
Table 2 Summary of immunohistochemical staining results of MUC1, MUC2, MUC5AC, MUC6, CDX2 in ampulla of Vater adenocarcinomas

\begin{tabular}{|c|c|c|c|c|c|c|}
\hline \multirow[t]{2}{*}{ Ampullary carcinomas } & \multirow{2}{*}{$\begin{array}{l}\text { Total cases } \\
53\end{array}$} & \multicolumn{5}{|c|}{ Positive cases $(\%)$} \\
\hline & & $\begin{array}{l}\text { MUC1 } \\
40(75)\end{array}$ & $\begin{array}{l}\text { MUC5AC } \\
23(43)\end{array}$ & $\begin{array}{l}\text { MUC6 } \\
21 \text { (39) }\end{array}$ & $\begin{array}{l}\text { MUC2 } \\
15(28)\end{array}$ & $\begin{array}{l}\text { CDX2 } \\
32(60)\end{array}$ \\
\hline \multicolumn{7}{|c|}{ Macroscopic classification } \\
\hline PA & 11 & $7(63)$ & $4(36)$ & $5(45)$ & $4(36)$ & $10(90)$ \\
\hline IA & 35 & $30(86)$ & $15(43)$ & $15(43)$ & $8(23)$ & $17(48)$ \\
\hline MT & 7 & $3(42)$ & $4(43)$ & $1(14)$ & $3(42)$ & $5(71)$ \\
\hline \multicolumn{7}{|l|}{ Histological type } \\
\hline $\mathrm{BP}$ & 30 & $29(97)$ & $17(57)$ & $13(43)$ & $4(13)$ & $9(30)$ \\
\hline I & 23 & $11(47)$ & $6(26)$ & $8(35)$ & $11(47)$ & $23(100)$ \\
\hline
\end{tabular}

$P A$ Periampullary, $I A$ intra-ampullary, $M T$ mixed type, $B P$ biliopancreatic type, $I$ intestinal type

out of the 30 BP-type ACs (Table 1), whereas seven cases (30.4\%) out of 23 intestinal-type ACs were associated with a BP pattern as a second component. Thirty-three tumors were moderately differentiated (grade 2), and 20 were poorly differentiated (grade 3 ). The $\mathrm{T}$ stage distribution was: 11 cases $(21 \%)$ at $\mathrm{T} 1,21(40 \%)$ at $\mathrm{T} 2,16(30 \%)$ at $\mathrm{T} 3$, and $5(9 \%)$ at $\mathrm{T} 4$. The pancreatic and choledochus resection margins were negative in all the cases. The posterior fat tissue was macroscopically negative in all cases but residual neoplastic disease (microscopically positive margin of resection: R1) was found in ten cases (19.2\%). A total of 924 regional lymph nodes were examined, (range 5-49, mean 18.8; median 14). Lymph node metastases were observed in 28 out of the $49(57 \%)$ cases for which the information was available (range 1-32, mean 4.7, median 2). The stage distribution was: 18 cases (37\%) at stage 1,26 $(53 \%)$ at stage $2,5(10 \%)$ at stage 3 .

Immunohistochemical and molecular study

The immunohistochemical expression of $\mathrm{CDX} 2$ and MUC1, MUC2, MUC5AC, and MUC6 apomucins was significantly different in intestinal and BP ACs. In particular, all intestinal ACs showed a nuclear immunoreactivity for CDX2 with 19 out of 23 cases demonstrating a high level of expression (score $3+$ in seven cases and $2+$ in 12 cases) compared with BP ACs exhibiting CDX2 immunoreactivity in nine out of $30(30 \%)$ of cases (score

Fig. 1 Histology and immunohistochemistry of grade II ampullary adenocarcinoma of biliopancreatic type (a) with MUC1 expression (b) and of grade II ampullary carcinoma of intestinal type (c) with MUC2 immunoreactivity (d). Original magnification $\times 200$ in (a), (b), (d); $\times 100$ in (c)
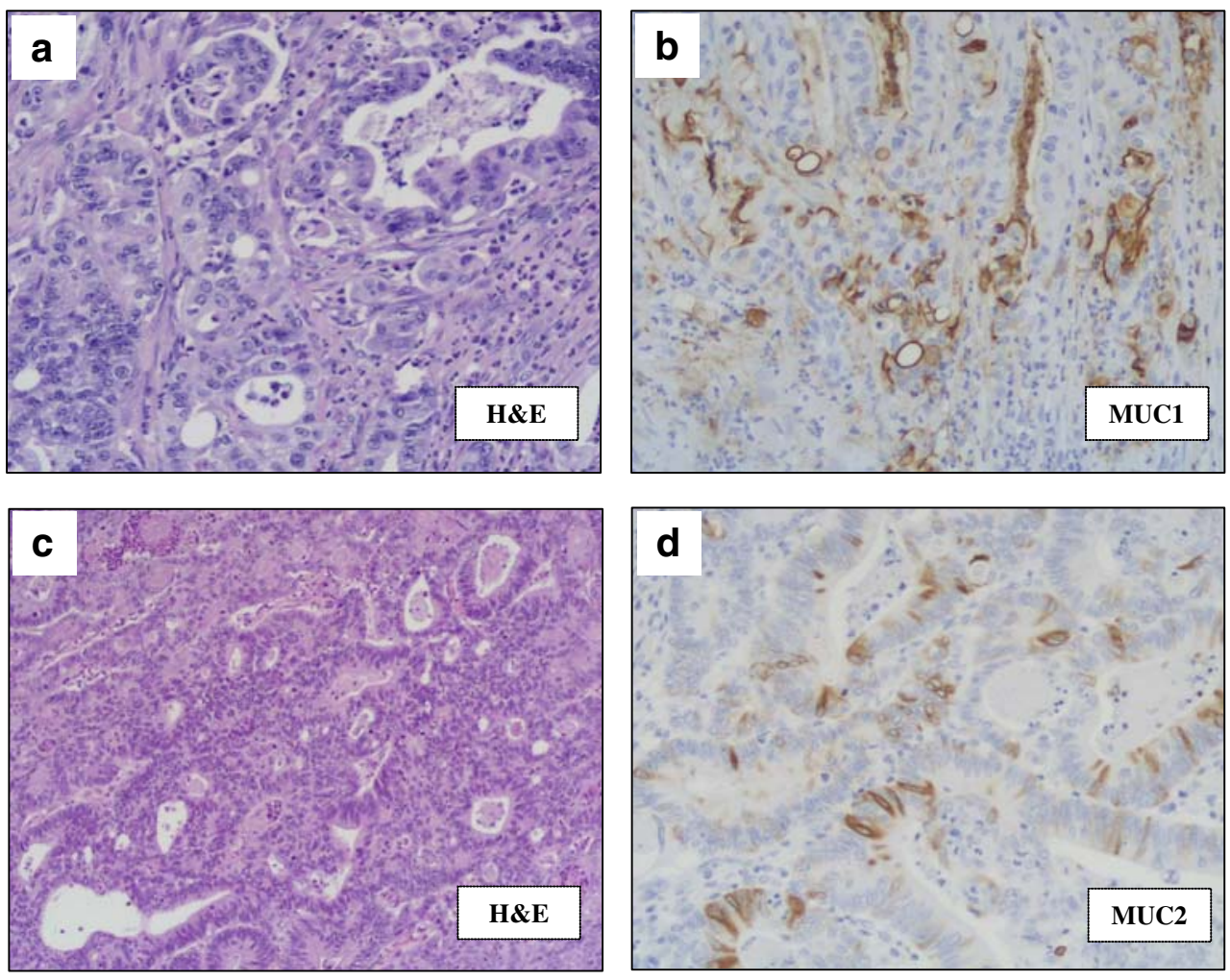
Table 3 Immunohistochemical expression of MMR proteins and mononucleotide instability in ampullary carcinomas with MSI in at least one locus

\begin{tabular}{|c|c|c|c|c|c|c|c|c|c|c|}
\hline \multirow[t]{2}{*}{$N^{\circ}$} & \multirow[t]{2}{*}{ Histology } & \multicolumn{4}{|c|}{ Immunohistochemical analysis } & \multicolumn{5}{|c|}{ MSI analysis } \\
\hline & & hMLH1 & hMSH2 & hMSH6 & hPMS2 & Bat-26 & Bat-25 & NR-21 & NR-22 & NR-24 \\
\hline 1 & AC-I & - & + & + & - & + & + & + & + & + \\
\hline 2 & AC-I, Muc & - & + & + & - & + & + & - & - & + \\
\hline 3 & AC-I, Muc & + & - & - & + & + & + & + & + & + \\
\hline 4 & AC-I, Med & + & - & - & + & + & + & + & + & + \\
\hline 5 & AC-I, Med & + & - & - & + & + & + & + & na & na \\
\hline 6 & AC-I, BP & + & + & + & + & + & + & - & - & - \\
\hline 7 & AC-I & + & + & + & + & - & + & - & - & - \\
\hline 8 & AC-I & + & + & + & + & - & + & - & - & - \\
\hline
\end{tabular}

$A C$-I Ampullary carcinoma of intestinal type, $M u c$ mucinous, $M e d$ medullary type, $B P$ biliopancreatic type, $M$ methylated, $U$ unmethylated, $n a$ not available

$2+$ in 3 cases and $1+$ in 6 cases; $p<0.000001)$. Notably, all but one of the BP ACs which were positive for CDX2 showed an intestinal and/or a mucinous component.

A nuclear immunoreactivity for CDX2 was found in 32 out of $53(60 \%)$ ACs with a significantly higher percentage of positive cases among PA and MT ACs compared with IA ACs $(p=0.028)$. Considering the grading of the tumors, CDX2 staining was found in 18 of $33(54.5 \%)$ moderately differentiated and, in 13 of $20(65 \%)$, poorly differentiated adenocarcinomas, but no significant correlation was observed.

The MUC1, MUC2, MUC5AC, MUC6 apomucins appeared to be expressed in $75,43,39$, and $28 \%$ of ACs, respectively. A significantly higher frequency of MUC1 and MUC5AC expression was detected in BP than in

a
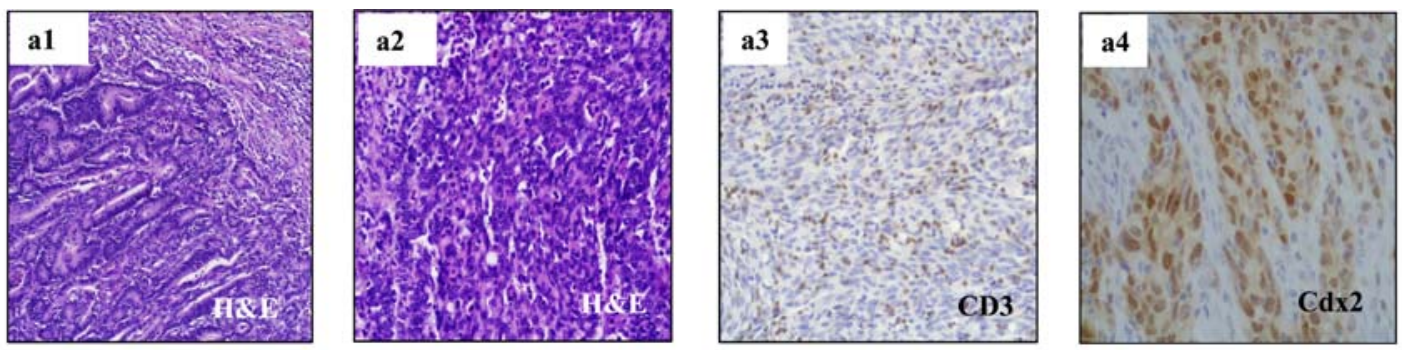

b

\section{b1) normal tissue}
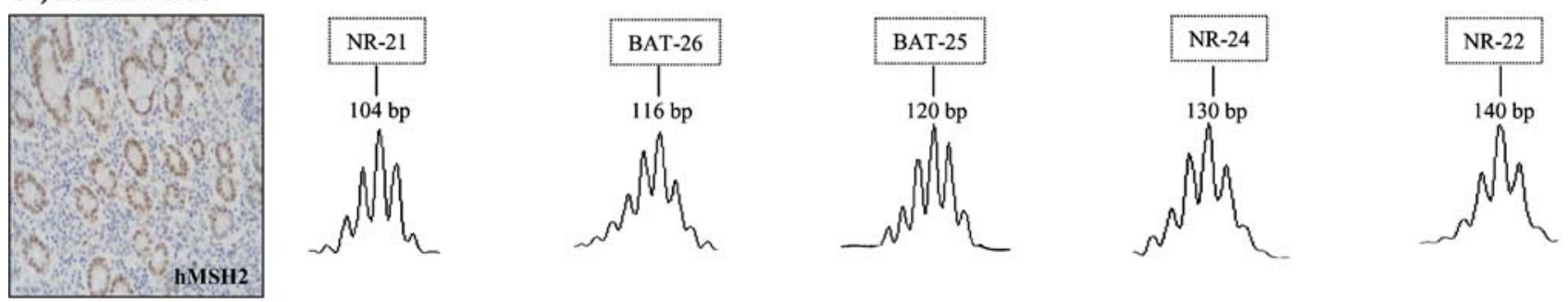

\section{b2) tumor}

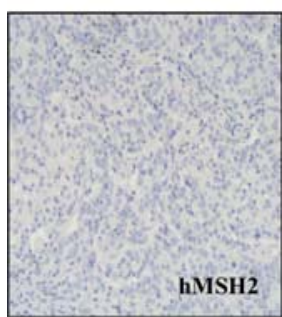

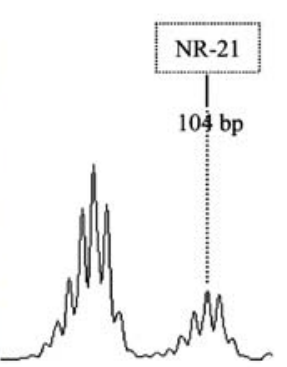
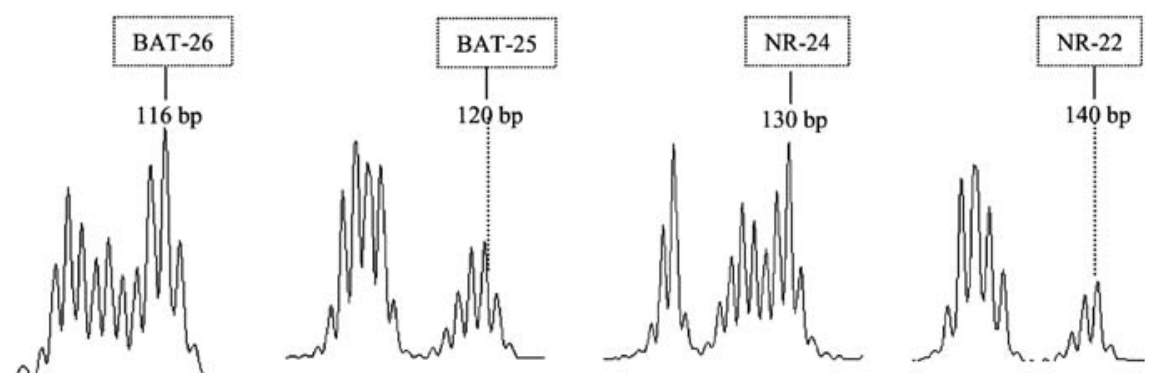

Fig. 2 Example of an intestinal-type AC (case 40) showing CD3 and CDX2 immunoreactivity (a) and loss of hMSH2 immunohistochemical expression and presence of MSI at all five mononucleotide markers examined (b) 
a
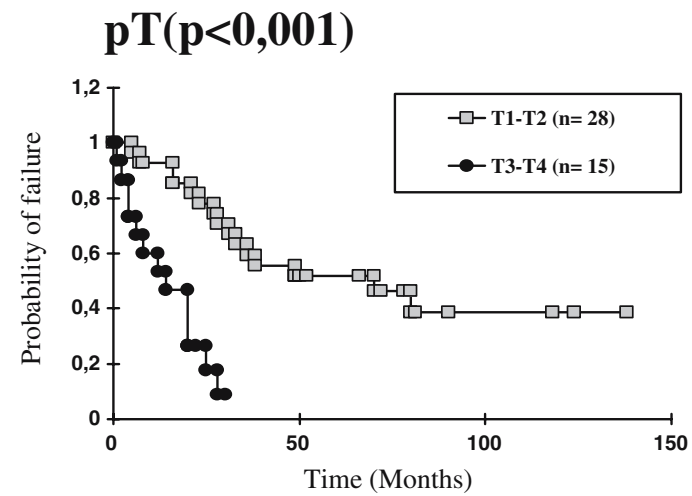

C Stage $(p=0,0003)$

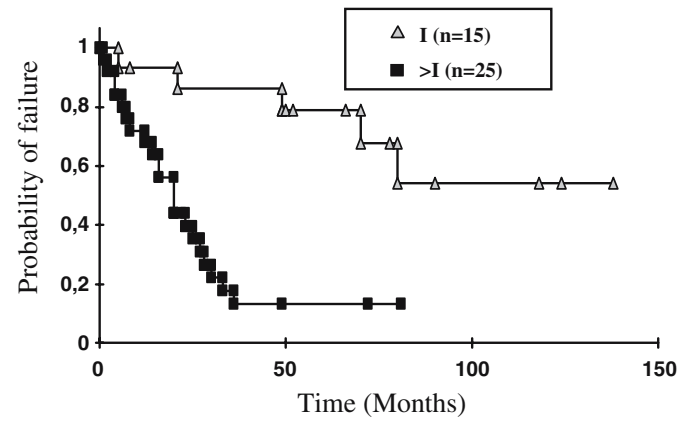

e $\quad \operatorname{Histology}(p=0,0804)$

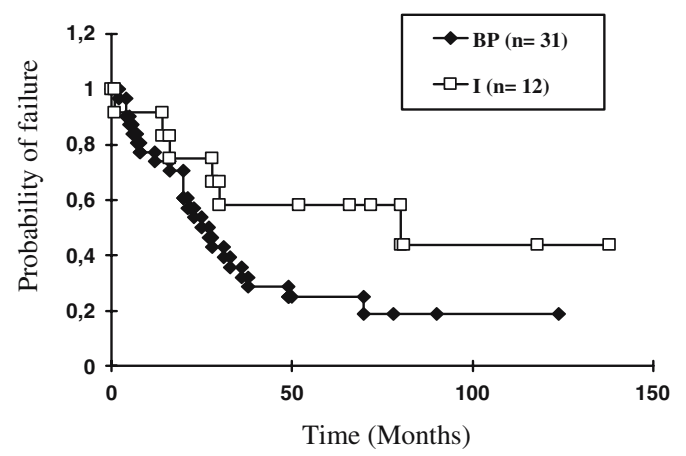

g CDX2 (cut off of $>10 \%$ cells) $(p=0,14)$

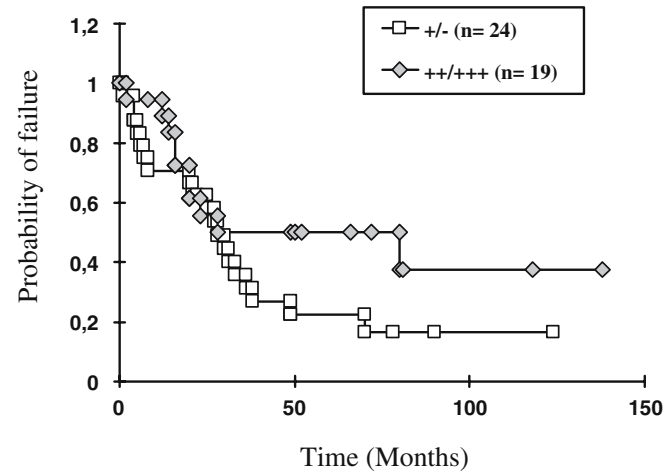

b $\quad \mathrm{pN}(\mathrm{p}=\mathbf{0 , 0 0 1 7})$

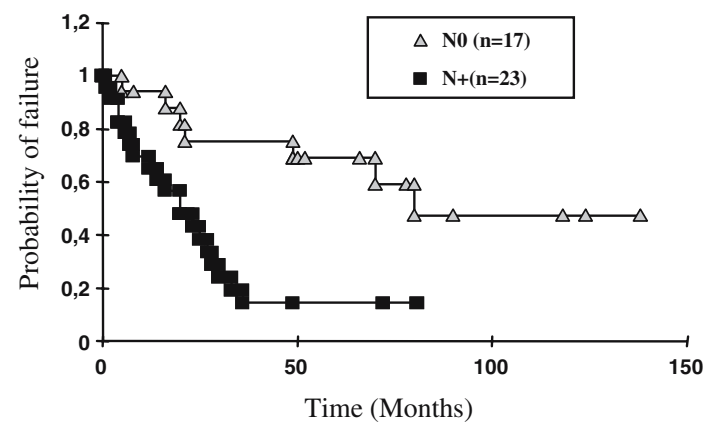

d

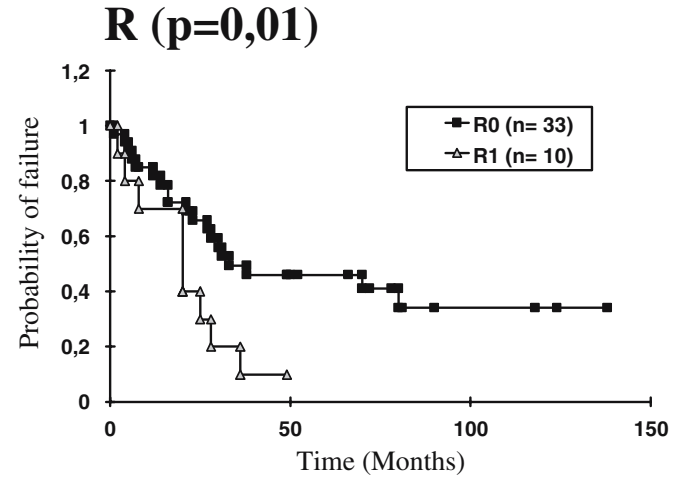

f

MSI status $(p=0,019)$

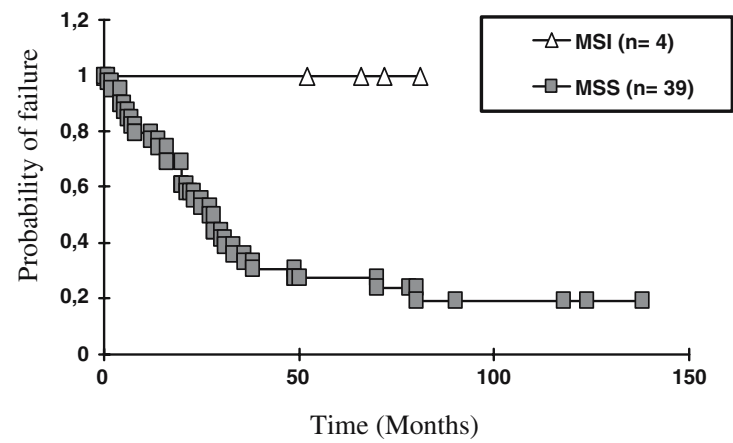

h CDX2 (cut off of $>50 \%$ cells $)(p=0,86)$

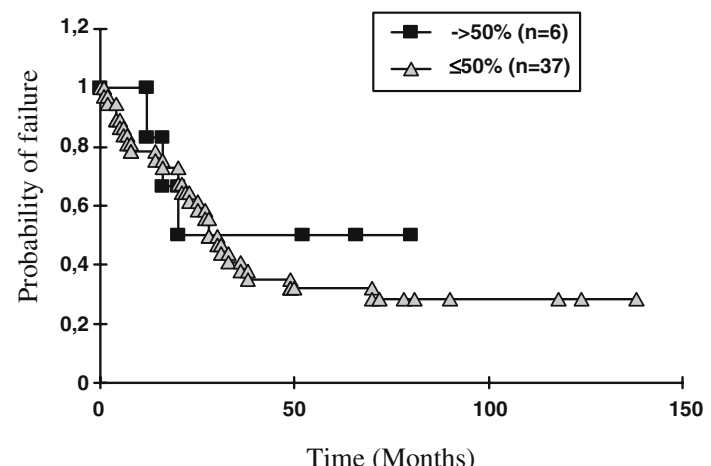

Fig. 3 Factors influencing survival in the univariate analysis were presence of microsatellite instability (f), clinical and pathological stage (c and a, respectively), lymph node metastases $(\mathbf{b}), R$ status $(\mathbf{d})$, histology (e), CDX2 immunoreactivity (g-h) 
Table 4 Results of the multivariate analysis interval, $S E$ standard error

\begin{tabular}{lllrl}
\hline Variable & HR & $95 \%$ CI & SE & $p$ value \\
\hline Histology & 0.73 & $0.2335-2.3$ & 0.58 & 0.5943 \\
CDX2 & 1.00 & $0.4143-2.4568$ & 0.45 & 0.9845 \\
MSI status & 0.00 & $0-1.7$ E34 & 45.77 & 0.8117 \\
$R$ status & 0.63 & $0.2528-1.5805$ & 0.46 & 0.3266 \\
Stage & 6.84 & $1.8756-25.0107$ & 0.66 & 0.0036 \\
\hline
\end{tabular}

Factors influencing survival in the univariate analysis were

intestinal ACs $(p<0.0001$ and $p<0.05$, respectively, for the two antibodies), whereas a significantly higher percentage of positive cases for MUC2 was found among intestinal ACs compared with BP ACs $(p<0.023)$. Moreover, a higher frequency of MUC1 immunoreactivity was detected in the IA cancers ( $86 \%$ of tumors) than in PA and MT ACs (63 and $42 \%$ of tumors, respectively). No other significant correlations were detected between the immunostaining results for the apomucins and macroscopic classification, histological type, or grading of ACs. MUC6 was rarely expressed in the ACs included in this study, and positive cases showed a focal immunoreactivity.

MSI was observed in five out of 53 ampullary ACs (9.5\%) showing allelic size variations in at least three of the five mononucleotide markers (Tables 1 and 3). The remaining tumors were classified as MSS, exhibiting an absence of MSI in all markers or the presence of allelic size variations at two loci (case $N^{\circ} 6$ in Table 3) or at one locus (cases $N^{\circ} 7$ and $N^{\circ} 8$ in Table 3). All five MSI tumors were intestinaltype ACs showing a mucinous component in two cases, a medullary component in two cases and a rich lymphoid infiltrate around the tumor in one case (Fig. 1). Moreover, all five MSI tumors showed a CDX2 immunoreactivity and a variable pattern of immunohistochemical expression of apomucins (Table 1).

To corroborate the MSI data, all eight ACs showing allelic size variations in at least one locus were analyzed for hMLH1, hMSH2, hMSH6, and PMS2 immunostaining (Table 3). All the five MSI ACs showed lack of the immunohistochemical expression of hMLH1/PMS2 proteins (cases 1 and 2) or of hMSH2/hMSH6 proteins (cases 3, 4, 5). By contrast, the three cases classified as MSS displayed an intense nuclear immunoreactivity for all MMR proteins. Figure 2 shows an example of the histological, immunohistochemical, and molecular profile of an AC with MSI.

\section{Survival analysis}

Follow-up data were available for 52 of the 53 patients with ACs. Nine patients were excluded from the survival analysis because they died within the first 60 days after tumor resection. Twenty-nine patients ( $67 \%$ of cases) died of disease after a median time of 24.8 months (range: 4 80 ), whereas 14 patients ( $33 \%$ of cases) are still alive after a median follow-up time of 71.2 months (range: 8-138).
(Fig. 3): tumor stage (median survival $>49$ months for T1-T2 vs 7 months for T3-T4, $p<0.001$ ), lymph node metastases (median survival $>52$ months for lymph node negative tumors vs 20 months for lymph node positive tumors, $p=$ 0.0017 ), $R$ status (median survival $>31$ months for complete tumor resection with grossly and microscopically negative margins vs 20 months for grossly negative but microscopically positive margins of resections), and presence of MSI (all patients with MSI tumors were alive with no evidence of disease after a median follow-up time of 69 months, $p=$ 0.019 ). Although the results were not statistically significant, a longer survival was correlated with the immunohistochemical expression of CDX2, using a nuclear labeling cut-off of $>10 \%$ cells $(p=0.14)$ as well as with an intestinal type of $\mathrm{AC}(p=0.08)$. No correlation with survival was observed even when considering different cut-offs for CDX2, including $\geq 25,30,40$, and $50 \%$ nuclear labeling. Finally, no other correlation was observed between patient outcome and clinicopathological parameters including macroscopic localization, size, and grade of the tumor.

A multivariate analysis was performed using the Cox proportional hazard model for all variables which were significant or near to statistical significance in the univariate analysis, and only stage was an independent prognostic factor (Table 4).

\section{Discussion}

ACs demonstrate a wide spectrum of biological behavior that probably reflects their different histogenesis, arising from one of the three types of epithelium that converge at this site (duodenal mucosa, pancreatic ductal epithelium, and biliary ductal epithelium). Currently, conflicting data have been reported about the frequency of the two major histological types of ACs (intestinal and BP type) due to the absence of reliable histomorphological or immunohistochemical markers for differential diagnosis [2, 20, 31]. Very recently, Hansel et al. [18] examined a large series of ACs demonstrating that CDX2 expression alone may identify intestinal-type tumors with good prognosis, suggesting that it may be used as an independent marker of outcome at this site.

In this work we evaluated the correlation between the histomorphological classification and the immunohisto- 
chemical expression of CDX2 and found a high specificity of the protein for intestinal-type ACs and for the minor intestinal and/or mucinous components of the BP-type ACs (Table 1). Intriguingly, a significantly higher percentage of CDX2 immunoreactive cases was observed among PA and MT ACs compared with IA ACs $(p=0.028)$ in line with the possible origin of these cancers in duodenal mucosa.

As regards the prognostic role of CDX2 expression, our results are in contrast with previously reported data by Hansel et al. [18] showing that CDX2 expression in ACs was the only independent predictor of survival in the multivariate analysis of their AC cases. In the present work, although CDX2 expression showed a trend towards a longer survival in the univariate analysis, no significant prognostic role as an independent marker was demonstrated for this variable in the multivariate analysis.

The univariate analysis showed that, microscopically positive margins of resection (R1), advanced clinical stages, and lymph node metastases were significantly related to a shorter survival, whereas the presence of MSI was a marker of a better outcome. In the multivariate analysis, stage group remained the only significant and independent prognostic factor of survival, as reported in other studies [4, 22, 24].

A widespread MSI as well as loss of the immunohistochemical expression of hMLH1/PMS2 or hMSH2/hMSH6 proteins were observed in five ACs $(9.5 \%$ of all cases, $22 \%$ of the intestinal ACs). All MSI ACs were of an intestinal type, and half were associated with a mucinous or with a medullary component. In one case, a rich lymphoid infiltrate around the tumor was observed. In addition, all MSI ACs were intensely CDX2-positive. This histopathological and immunohistochemical profile appears to be similar to that of gastric and colorectal MSI cancers $[6,9$, $13,19]$ and confirms the notion that MSI is a peculiar feature of a consistent percentage of intestinal-type ACs [27]. Until now, few and controversial data have been reported about the frequency of a MSI in ACs [1, 25, 29] probably due to different criteria for tumor selection, including cases of not unequivocally ampullary origin and ACs of different types. Our results regarding MSI ACs are comparable with those reported by Achille et al. [1] that indicated a $20 \%$ rate of MSI ACs in a series of 25 cases and reported a significant association between MSI and longer patient survival in the univariate analysis.

Finally, differences in the mucin distribution between BP and intestinal ACs were observed. The coexpression of MUC1 and MUC5AC appears to be a peculiar feature of BP $\mathrm{ACs}$, whereas a strong production of MUC2 is associated with an intestinal histology and a CDX-2 positive immunophenotype. These data are consistent with the findings reported by Chu et al. [10] and suggest that the two main immunoprofiles mentioned above are helpful in the differential diagnosis between intestinal and BP types of ACs. However, the composite pattern of apomucin expression often observed in ACs does not allow a clear-cut classification of the two major histological types and suggests only an ancillary use of apomucins in this differential diagnosis. Furthermore, no significant correlation between mucin immunoprofile and survival was observed. Similar results have been reported by using other immunohistochemical markers such as cytokeratin 7 and cytokeratin 20 [10,31].

In conclusion, this study shows a comprehensive analysis of the main clinicopathological, immunohistochemical, and molecular parameters suggested to have an impact on survival of patients with ACs and proves that a precise stage assessment remains the only independent predictor of survival. The extent of resection appears to be prognostically of great significance, although it is not part of the TNM staging as well as negative lymph nodes, and presence of MSI are significantly correlated with improved outcome for the patients.

Although precise histological criteria and the immunohistochemical expression of apomucines and CDX2 are helpful in the classification of the two major histological types of ACs, no significant correlation between intestinal profiles and improved survival is observed.

\section{References}

1. Achille A, Biasi MO, Zamboni G, Bogina G, Iacono C, Talamini G, Capella G, Scarpa A (1997) Cancers of the papilla of Vater: mutator phenotype is associated with good prognosis. Clin Cancer Res 3:1841-1847

2. Albores-Saavedra J, Murakata L, Krueger JE, Henson DE (2000) Noninvasive and minimally invasive papillary carcinomas of the extrahepatic bile ducts. Cancer 89:508-515

3. Albores-Saavedra J, Hoang MP, Murakata LA, Sinkre P, Yaziji H (2001) Atypical bile duct adenoma, clear cell type: a previously undescribed tumor of the liver. Am J Surg Pathol 25:956-960

4. Allema JH, Reinders ME, van Gulik TM, van Leeuwen DJ, Verbeek PC, de Wit LT, Gouma DJ (1995) Results of pancreaticoduodenectomy for ampullary carcinoma and analysis of prognostic factors for survival. Surgery 117:247-253

5. Barbareschi M, Murer B, Colby TV, Chilosi M, Macri E, Loda M, Doglioni C (2003) CDX-2 homeobox gene expression is a reliable marker of colorectal adenocarcinoma metastases to the lungs. Am J Surg Pathol 27:141-149

6. Benatti P, Gafa R, Barana D, Marino M, Scarselli A, Pedroni M, Maestri I, Guerzoni L, Roncucci L, Menigatti M, Roncari B, Maffei S, Rossi G, Ponti G, Santini A, Losi L, Di Gregorio C, Oliani C, Ponz de Leon M, Lanza G (2005) Microsatellite instability and colorectal cancer prognosis. Clin Cancer Res $11: 8332-8340$

7. Bonner CA, Loftus SK, Wasmuth JJ (1995) Isolation, characterization, and precise physical localization of human CDX1, a caudal-type homeobox gene. Genomics 28:206-211

8. Chiaravalli AM, Furlan D, Facco C, Tibiletti MG, Dionigi A, Casati B, Albarello L, Riva C, Capella C (2001) Immunohistochemical pattern of hMSH2/hMLH1 in familial and sporadic colorectal, gastric, endometrial and ovarian carcinomas with instability in microsatellite sequences. Virchows Arch 438:39-48 
9. Chong JM, Fukayama M, Hayashi Y, Takizawa T, Koike M, Konishi M, Kikuchi-Yanoshita R, Miyaki M (1994) Microsatellite instability in the progression of gastric carcinoma. Cancer Res 54:4595-4597

10. Chu PG, Schwarz RE, Lau SK, Yen Y, Weiss LM (2005) Immunohistochemical staining in the diagnosis of pancreatobiliary and ampulla of Vater adenocarcinoma: application of CDX2, CK17, MUC1, and MUC2. Am J Surg Pathol 29:359-367

11. Cubilla AL, Fitzgerald PJ (1980) Surgical pathology aspects of cancer of the ampulla-head-of-pancreas region. Monogr Pathol 21:67-81

12. Dawson PJ, Connolly MM (1989) Influence of site of origin and mucin production on survival in ampullary carcinoma. Ann Surg 210:173-179

13. dos Santos NR, Seruca R, Constancia M, Seixas M, SobrinhoSimoes M (1996) Microsatellite instability at multiple loci in gastric carcinoma: clinicopathologic implications and prognosis. Gastroenterology 110:38-44

14. Drummond F, Putt W, Fox M, Edwards YH (1997) Cloning and chromosome assignment of the human CDX2 gene. Ann Hum Genet 61:393-400

15. Furlan D, Cerutti R, Genasetti A, Pelosi G, Uccella S, La Rosa S, Capella C (2003) Microallelotyping defines the monoclonal or the polyclonal origin of mixed and collision endocrine-exocrine tumors of the gut. Lab Invest 83:963-971

16. Gurbuz Y, Kloppel G (2004) Differentiation pathways in duodenal and ampullary carcinomas: a comparative study on mucin and trefoil peptide expression, including gastric and colon carcinomas. Virchows Arch 444:536-541

17. Albores-Saavedra J (2001) Carcinoma of the gallbladder and extrahepatic bile ducts. In: Hamilton SR and Aaltonen LA (eds) Tumor of the digestive system. Lyon: IARC Press, pp 206-214

18. Hansel DE, Maitra A, Lin JW, Goggins M, Argani P, Yeo CJ, Piantadosi S, Leach SD, Biankin AV (2005) Expression of the caudaltype homeodomain transcription factors CDX $1 / 2$ and outcome in carcinomas of the ampulla of Vater. J Clin Oncol 23:1811-1818

19. Kim H, Jen J, Vogelstein B, Hamilton SR (1994) Clinical and pathological characteristics of sporadic colorectal carcinomas with DNA replication errors in microsatellite sequences. Am J Pathol 145:148-156

20. Kimura W, Futakawa N, Yamagata S, Wada Y, Kuroda A, Muto T, Esaki Y (1994) Different clinicopathologic findings in two histologic types of carcinoma of papilla of Vater. Jpn J Cancer Res 85:161-166

21. Kitamura H, Yonezawa S, Tanaka S, Kim YS, Sato E (1996) Expression of mucin carbohydrates and core proteins in carcinomas of the ampulla of Vater: their relationship to prognosis. Jpn J Cancer Res 87:631-640

22. Klempnauer J, Ridder GJ, Pichlmayr R (1995) Prognostic factors after resection of ampullary carcinoma: Multivariate survival analysis in comparison with ductal cancer of the pancreatic head. Br J Surg 82:1686-1691

23. Matsubayashi H, Watanabe H, Yamaguchi T, Ajioka Y, Nishikura K, Kijima H, Saito T (1999) Differences in mucus and K-ras mutation in relation to phenotypes of tumors of the papilla of Vater. Cancer 86:596-607

24. Neoptolemos JP, Talbot IC (1988) Ampullary carcinoma. Br J Surg 75:829-830

25. Park S, Kim SW, Kim SH, Darwish NS, Kim WH (2003) Lack of microsatellite instability in neoplasms of ampulla of Vater. Pathol Int 53:667-670

26. Paulsen FP, Varoga D, Paulsen AR, Corfield A, Tsokos M (2006) Prognostic value of mucins in the classification of ampullary carcinomas. Human Pathol 37:160-167

27. Scarpa A, Di Pace C, Talamini G, Falconi M, Lemoine NR, Iacono C, Achille A, Baron A, Zamboni G (2000) Cancer of the ampulla of Vater: Chromosome 17p allelic loss is associated with poor prognosis. Gut $46: 842-848$

28. Suraweera N, Duval A, Reperant M, Vaury C, Furlan D, Leroy K, Seruca R, Iacopetta B, Hamelin R (2002) Evaluation of tumor microsatellite instability using five quasimonomorphic mononucleotide repeats and pentaplex PCR. Gastroenterology 123:18041811

29. Suto T, Habano W, Sugai T, Uesugi N, Kanno S, Saito K, Nakamura S (2001) Infrequent microsatellite instability in biliary tract cancer. J Surg Oncol 76:121-126

30. Werling RW, Yaziji H, Bacchi CE, Gown AM (2003) CDX2, a highly sensitive and specific marker of adenocarcinomas of intestinal origin: an immunohistochemical survey of 476 primary and metastatic carcinomas. Am J Surg Pathol 27:303-310

31. Zhou H, Schaefer N, Wolff M, Fischer HP (2004) Carcinoma of the ampulla of Vater: comparative histologic/immunohistochemical classification and follow-up. Am J Surg Pathol $28: 875-882$ 